\title{
Age Group Estimation Using Face Angle
}

\author{
Ranjan Jana ${ }^{1}$, Harekrishna Pal ${ }^{1}$, Amrita Roy Chowdhury ${ }^{2}$ \\ ${ }^{I}$ (Dept. of MCA, RCC Institute of Information Technology, India) \\ ${ }^{2}$ (Dept. of MCA, Academy of Technology, India)
}

\begin{abstract}
Recognition of the most facial variations, such as identity, expression and gender, has been extensively studied. Automatic age estimation has rarely been explored. With age progression of a human the face angle changes. This paper concerns with providing a methodology to estimate age groups using face features. The proposed method is based on the face triangle which has three coordinate points between left eye ball, right eyeball and mouth point. The face angle between left eyeball, mouth point and right eyeball estimates the age of a human. However, very few studies have been done on age classification or age estimation. This paper proves that face angle can estimate and classify human age according to face features extracted from human facial images. Age ranges are classified into five categories. Those are child (up to 17 years), young (18 to 25 years), adult (26 to 35 years), middle aged (36 to 45 years) and old (more than 45 years). The obtained results were significant. This paper can be used for predicting future faces, classifying gender, and expressions from facial images.
\end{abstract}

Keywords: Age estimation, Eyeball detection, Face angle, Face triangle, Mouth point detection

\section{Introduction}

Face recognition is an important area of biometrics which is of great use in our daily life. Traditionally, it uses for identification of documents such as land registration, passports, driver's licenses, recognition of a human in a security area. Face images are being increasingly used as additional means of authentication in applications of high security zone [1]. But with age progression the facial features changes and the database needs to be updated regularly which is a tedious task. So we need to address the issue of facial aging and come up with a mechanism that identifies a person in spite of the aging [2]. Traditional, Face Recognition includes methods like Eigen face or principal component analysis (PCA), fisherface or linear discriminate analysis (LDA) etc. These techniques [3, 4] extract facial features from an image and using them perform search in the face database for images with matching features. 3-D technique uses 3-D sensors to capture information about the shape of a face $[5,6]$. This information is then used to identify distinctive features on the surface of a face, such as the contour of the eye sockets, nose, and chin. This technique is robust to changes in lighting and viewing angles. Skin texture analysis technique [7,8] uses the visual details of the skin, as captured in standard digital or scanned images, and turns the unique lines, patterns, and spots apparent in a person's skin into a mathematical space.

In human computer interaction, aging effects in human faces has been studied from two main reasons: (1) automatic age estimation for face image classification and (2) automatic age progression for face recognition. Kwno et al. [1] developed a system to classify face images into one of three age groups: infants, young adults and senior adults. They extracted key landmarks from face images and calculated distances between those landmarks. Then ratios of those distances were used to classify face images as that of infants or adults. They also proposed methods for wrinkle detection in predetermined regions in face images to further classify adult images into young adults and senior adults. The first real human age estimation theory was proposed Lanitis et al. $[9,10]$. They proposed methods to imitate aging effects on face images. They developed an aging function (quadratic function) based on a parametric model of face images and performed tasks such as automatic age estimation, face recognition across age progression. They only considered database having face images of individuals less than 30 years of age.

N.Ramanathan and R.Chellappa $[2,11]$ developed a Bayesian age difference classifier that classifies face images of individuals based on age differences and performs face verification across age progression. They used coordinate transformation and deformation of local facial feature landmarks. But males and females may have different face aging patterns depending on nature effects. Geng et al. [12] proposed the AGES (Aging pattern Sub-space) method for automatic age estimation. They model the aging pattern in a 2D sub-space and then for an unseen face image, they construct the face and determine the age. Anil K. Jain [13] proposed a 3D aging modeling technique which automatically generates some missing images in different age groups. It learnt aging pattern based on PCA coefficients in separated 3D space and texture given 2D database. $[14,15]$ proposed that the frontal face view form an isosceles triangle combining the two eyes and one mouth. This isosceles triangle is quite useful for face recognition. From careful observation we conclude that this face triangle is unique for every person and this face triangle can be used for face recognition with age. 
In this paper a novel and effective age estimation using face triangle from human face image are proposed. For better performance, the face images of different ages are grouped into five age range: $1^{\text {st }}$ group from age 1 to 17 years as childhood, $2^{\text {nd }}$ group from age 18 to 25 years as young, $3^{\text {rd }}$ group from age 26 to 35 years as adult, $4^{\text {th }}$ group from age 36 to 45 years as middle aged and, $5^{\text {th }}$ group more than 45 years. This paper reviews several age range estimation from human face images and is organized as follows. Section II describes implementation details. Proposed algorithm for age range estimation is mentioned in section III. Experimented results are mentioned in section IV. Finally, the conclusions are in section V.

\section{Implementation}

There are several face detection and age group estimation have been proposed [1]-[15]. This paper proposed a novel and effective age estimation using face triangle from human face image as shown in Fig. 1. After detecting the face area, the eye area has been detected. The iris of the eye appears to be the darkest area. So, we detect right and left eyeball by searching from the eye area. After that mouth point has been detected from the face area. Now, we can form a triangle called face triangle with three coordinate points left eyeball, mouth point, and right eyeball as shown in Fig. 1. The angle between right eyeball, mouth point, and left eyeball is called face angle. With age progression of a human the face angle changes. So, by calculating the face angle we can estimate the age group.

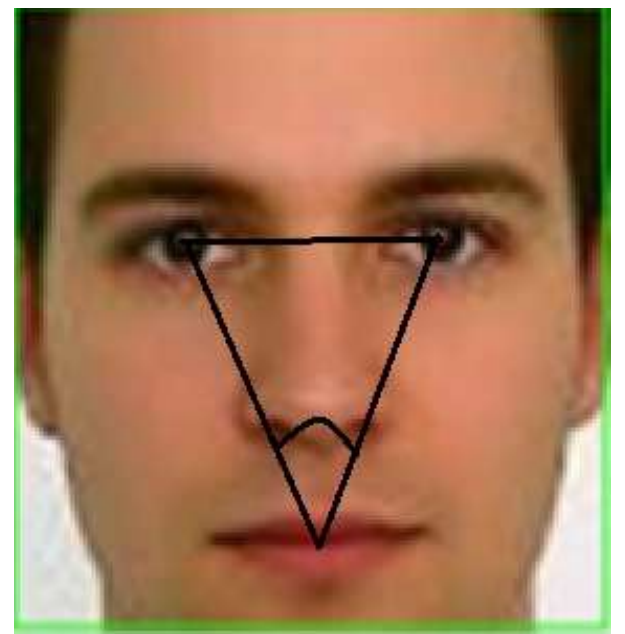

Figure 1: Face image with face triangle and face angle

\section{Algorithm For Age Group Estimation}

For age group estimation the following algorithm has been proposed. This algorithm uses some coordinate point that is shown in Fig. 2.

Step 1: Detect the rectangular face area from input face image using Matlab in-built object function. If number of detected face is more than one, an error message will be displayed.

Step 2: Crop the detected rectangular face area and detect the significance eye-region using Matlab in-built object function.

Step 3: The cropped rectangular face image is histogram equalized and then converted into binary image.

Step 4: The binary image of face is divided horizontally into two parts. Upper part that contains two eyes is denoted by $\mathrm{U}_{\text {PART }}$ and lower part that contains mouth is denoted by $\mathrm{L}_{\mathrm{PART}}$.

Step 5: Divide $U_{\text {PART }}$ vertically into two parts. One part that contains right eye is denoted by $R_{\text {EYE }}$ and other part that contains left eye is denoted by $\mathrm{L}_{\mathrm{EYE}}$.

Step 6: Find the row number R1 with minimum row sum of gray level in $\mathrm{U}_{\mathrm{PART}}$. Find the column numbers $\mathrm{C} 1$ and $\mathrm{C} 2$ with minimum column sum of gray level in $\mathrm{R}_{\mathrm{EYE}}$ and $\mathrm{L}_{\mathrm{EYE}}$. So, $(\mathrm{R} 1, \mathrm{C} 1)$ coordinate represents middle point of right eyeball and $(\mathrm{R} 1, \mathrm{C} 2)$ coordinate represents middle point of left eyeball.

Step 7: Find the row number R2 with minimum row sum of gray level in $\mathrm{L}_{\mathrm{PART}}$. So, R2 row represents the mouth row.

Step 8: Calculate the midpoint $\mathrm{C} 3$ of two eye ball. So, $\mathrm{C} 3=(\mathrm{C} 1+\mathrm{C} 2) / 2$ and the coordinate $(\mathrm{R} 2, \mathrm{C} 3)$ is middle point of mouth.

Step 9: Draw a triangle by three coordinate points left eyeball (R1, C1), right eyeball (R1, C2) \& mouth point (R2, C3). 
Step 10: Calculate slope (m1) of triangle sides from mouth point (R2, C3) to right eyeball (R1, C1) and slope (m2) of triangle sides from mouth point $(\mathrm{R} 2, \mathrm{C} 3)$ to left eyeball $(\mathrm{R} 1, \mathrm{C} 2)$.

Step 11: Find the face angle (A) using formula: $\quad A=\tan ^{-1}((m 1-m 2) /(1+m 1 * m 2))$

Step 12: Determine age group based on the face angle (A) as follows:

\begin{tabular}{|c|c|}
\hline $\begin{array}{c}\text { Face angle in degrees } \\
\text { (A) }\end{array}$ & Age group in years \\
\hline$<44$ & $<18$ \\
\hline 44 to 48 & 18 to 25 \\
\hline 49 to 54 & 26 to 35 \\
\hline 55 to 60 & 36 to 45 \\
\hline$>60$ & $>45$ \\
\hline
\end{tabular}

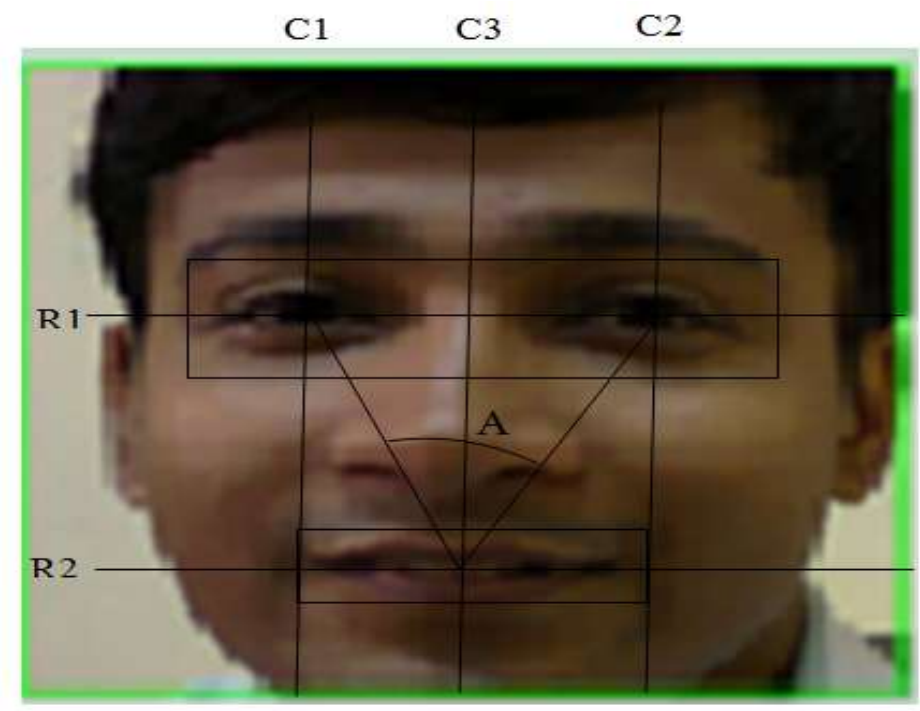

Figure 2: Face image with different coordinate points

\section{Experimental Results}

This section introduces the experimental results. The result of the above algorithm is shown step by step in Fig. 3.

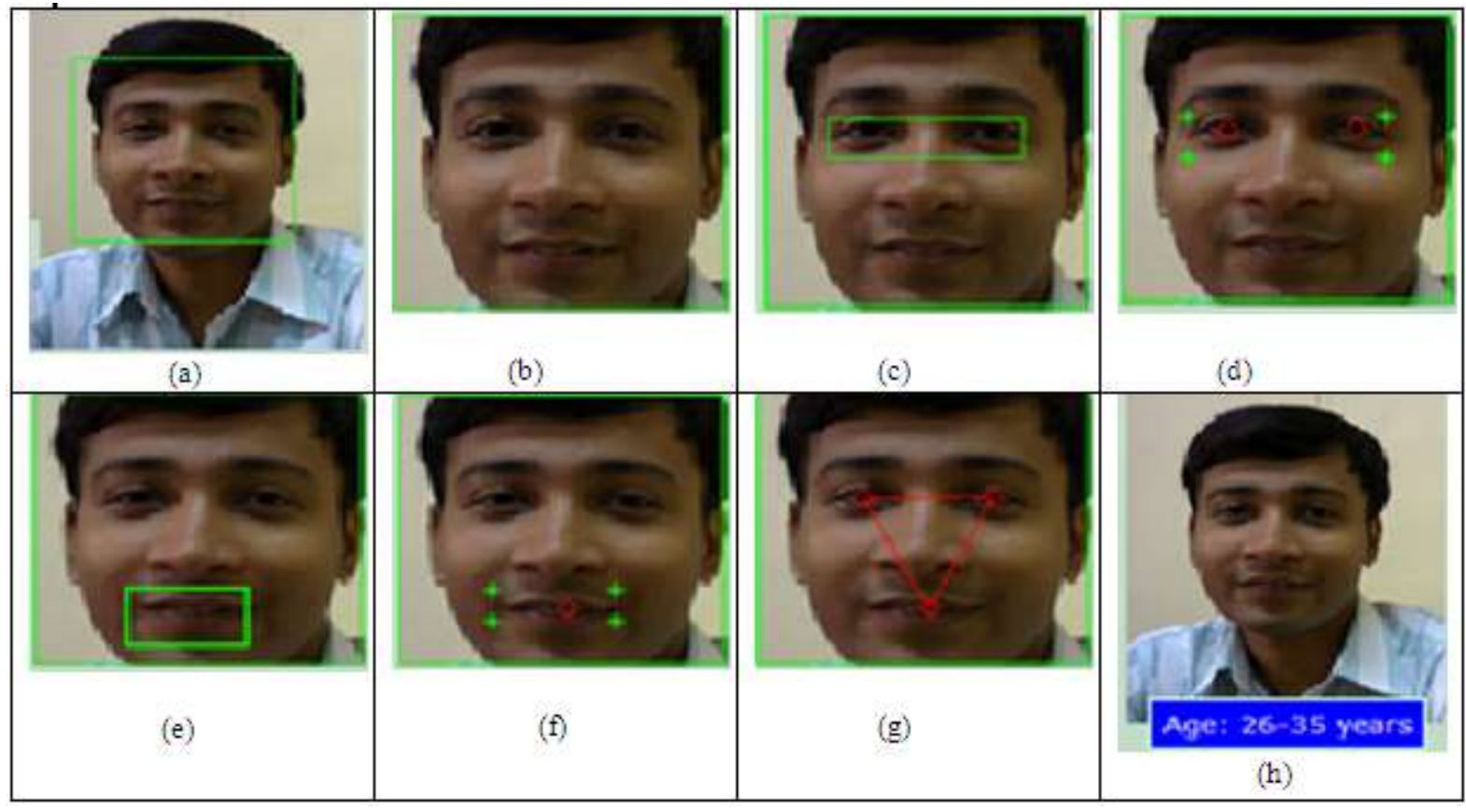


Figure 3: (a) Face detected image, (b) Cropped image, (c) Eye detected image, (d) Eyeball detected image, (e) Mouth detected image, (f) Mouth point detected image, (g) Face triangle and angle generated image, (h) Age estimated image

In Fig. 4, we have taken face image of a boy with nine years old. The output shows that the estimated age is less than eighteen years which is a correct estimation. In Fig. 5, we have taken face image of a boy with twenty seven years old. The output shows that the estimated age range is 26 to 35 years which is also a correct estimation. In Fig. 6 to Fig. 8, we have taken face images with different ages and the results are correct. But in Fig. 9, we have taken an image with three faces and the output generates a message "More than one face has been detected" which is also a correct result.

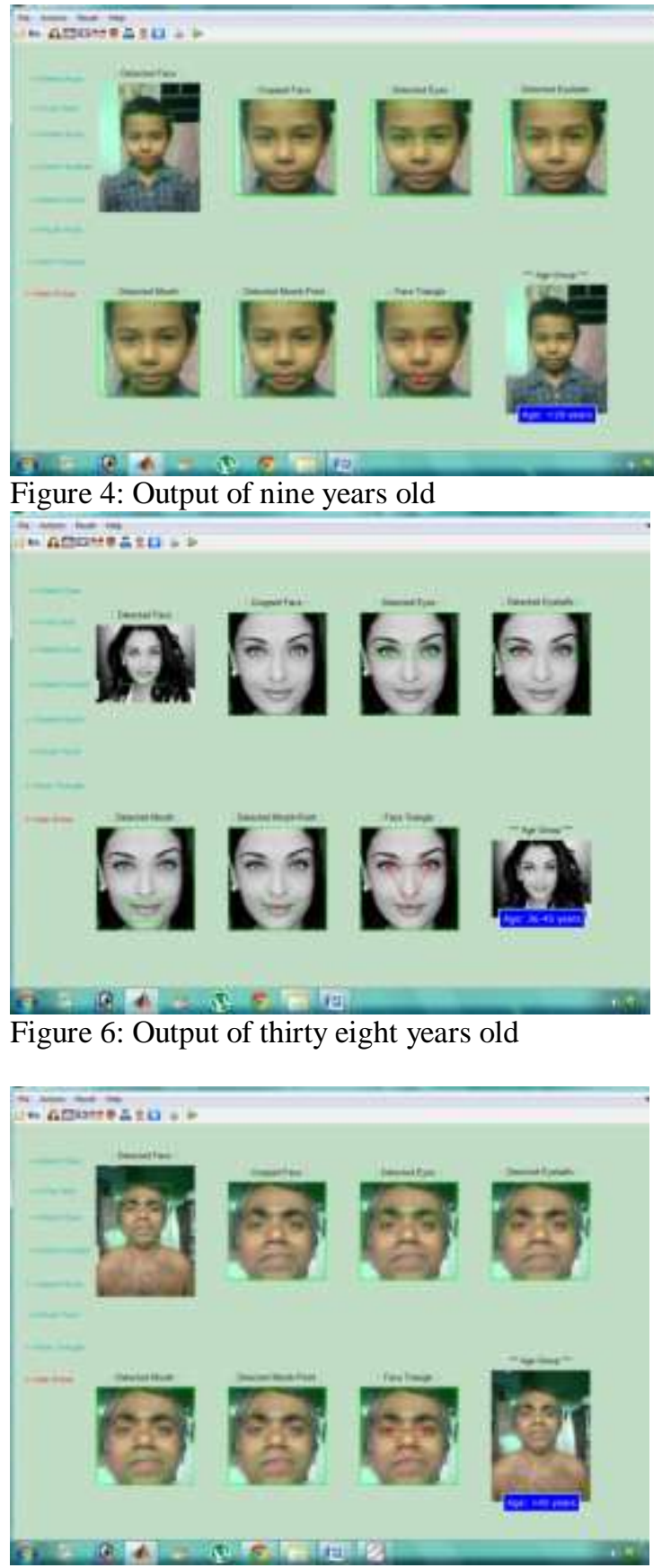

Figure 8: Output of sixty years old

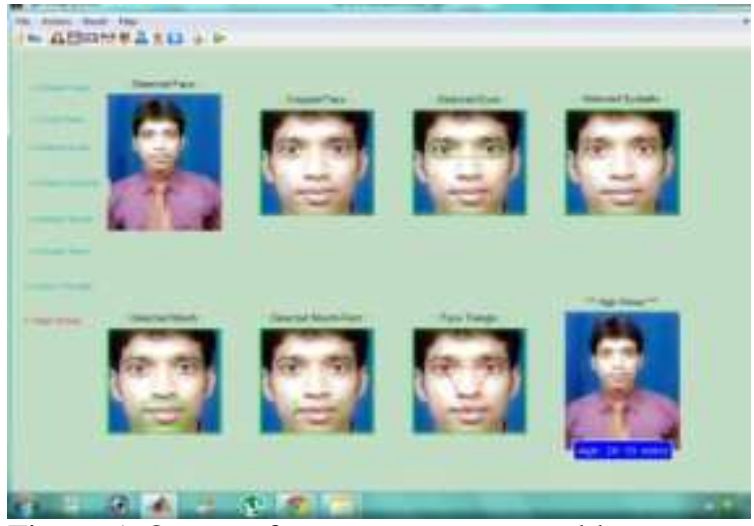

Figure 5: Output of twenty seven years old

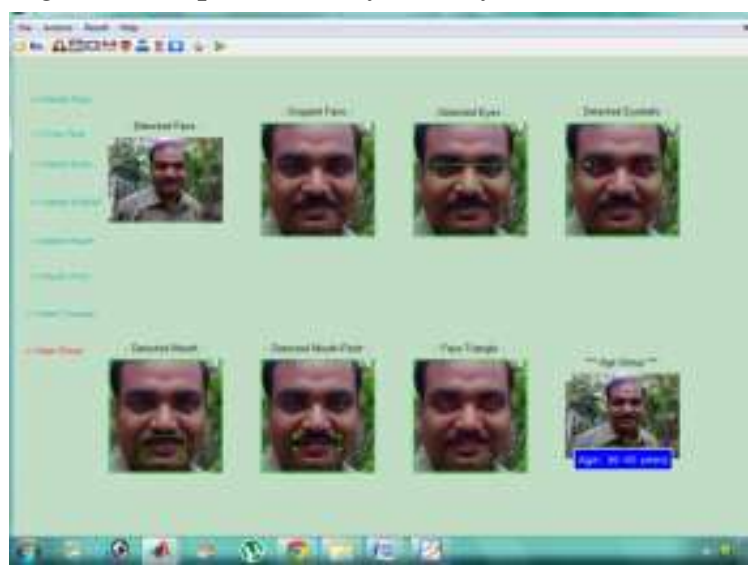

Figure 7: Output of thirty nine years old

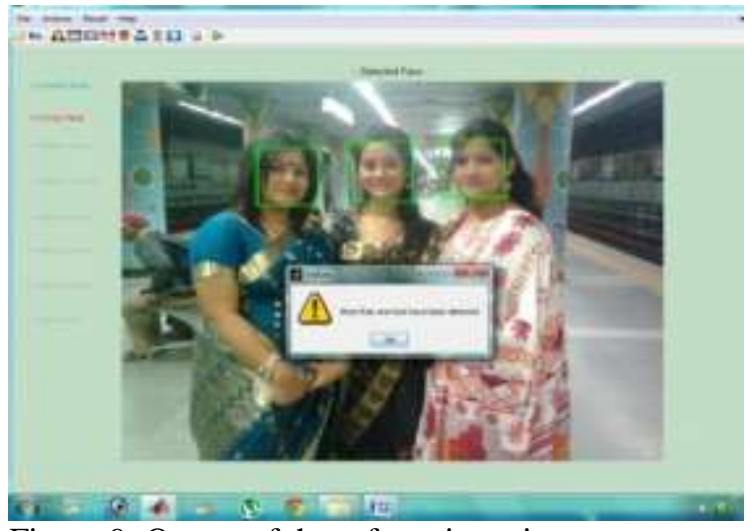

Figure 9: Output of three faces in an image

\section{Conclusion}

In this paper a method for age group estimation is thoroughly described. As face changes with age, it is very difficult to update periodically the databases where face recognition is very important. So the proposed technique provides a robust method that verifies the identity of individuals from a pair of age separated face 
images. It is also seen that in the age group 1-25 the structural changes in face are maximum. For proper eye and eyeball detection, face in the image should be without spectacle. Image should be of a straight frontal face. Image should contain single human face only. This paper works with $85 \%$ accuracy and some time generate erroneous outputs. But the method shows some difficulties in detecting the facial components if face image is not frontal image. So, there seems to be a definite possibility for further extension of the work which includes extracting more feature points such as base angle of the isosceles face triangle, face isosceles triangle in between two eyes and nose tip can improve accuracy of our match. By improving the implementation of the paper, the age range can be further narrowed.

\section{Acknowledgements}

The authors are grateful to Sudeshna Poddar and Payel Bhattacharya for their help and support to improve this paper.

\section{References}

[1] Y.H.Kwno and N.daVitoria Lobo, “Age Classification from Facial Images," Computer Vision and Image Understanding, vol.74, no.1, pp.1-21, 1999.

[2] N.Ramanathan and R. Chellappa, "Face verification across age progression," in Proc. IEEE Conf. Computer Vision and Pattern Recognition, San Diego, CA, 2005, vol.2, pp.462-469.

[3] P. N. Belhumeur, J. P. Hespanha, and D. J. Kriegman. Eigenfaces vs. Fisherfaces: Recognition using class specific linear projection. IEEE Transactions on Pattern Analysis and Machine Intelligence, 19(7):711-720, July 1997.

[4] M. A. Turk and A. P. Pentland. Eigenfaces for recognition. Journal of Cognitive Neuroscience, 3(1): 71-86, 1991.

[5] V. Blanz and T. Vetter. Face recognition based on fitting a 3D morphable model. IEEE Transactions on Pattern Analysis and Machine Intelligence, 25(9): 1063 -1074, September 2003.

[6] R. Kimmel A. M. Bronstein, M. M. Bronstein. Three-dimensional face recognition. Intl. Journal of Computer Vision, 64(1):5-30, August 2005.

[7] B.D., Zarit, B.J., Super, AND F.K.H. Quek, "Comparison of five color models in skin pixel classification”. In Int. Workshop on Recognition, Analysis, and Tracking of Faces and Gestures in Real-Time Systems, pages 58-63, Corfu, Greece, Sept. 1999.

[8] R.L., Hsu, M., Abdel-Mottaleb, and A.K.Jain, "Face detection in color images". IEEE Trans. on Pattern Analysis and Machine Intelligence, 24(5):696-706, May 2002

[9] A.Lanitis and C.J.Taylor, "Towards Automatic Face Identification Robust to Ageing Variation," IEEE Trans. on Pattern Analysis and Machine Intelligence, vol.24, no.24, p.442-455, 2002.

[10] A.Lanitis, C.Draganova, and C.Christodoulou, "Comparing different classifiers for automatic age estimation," IEEE Trans.Syst.Man, Cybern.B, Cybern, vol34, no.1, pp.621-628, Feb.2004.

[11] N.Ramanathan and R. Chellappa, "Modelling Age Progression in young faces," in Proc. IEEE Conf. Computer Vision and Pattern Recognition (CVPR), vol.1, pp.387-394, 2006.

[12] X.Geng, Z.H. Zhou, and K. Smith-Miles, “Automatic age estimation based on facial aging patterns," IEEE Trans. on Pattern Analysis and Machine Intelligence, vol.29, pp.2234-2240, 2007.

[13] Anil K.Jain, “Age Invariant Face Recognition,” IEEE Trans. on Pattern Analysis and Machine Intelligence.2010

[14] Chiunhsiun Lin, Kuo-Chin Fan, "Triangle-based approach to the detection of human face," Pattern Recognition Journal Society, vol.34, pp.1271-1284, 2001.

[15] Hiranmoy Roy, Debotosh Bhattacherjee, Mita Nasipuri, Dipak Kumar Basu, “Age Range Estimation from Human Face Images Using Face Triangle Formation" International Journal of Research and Reviews in Information Sciences (IJRRIS), Vol. 2, No. 1, pp.155-160, March 2012. 